Tecno Lógicas

ISSN 0123-7799

Vol. 17 , No. 32 , pp. $75-84$

Enero-junio de 2014
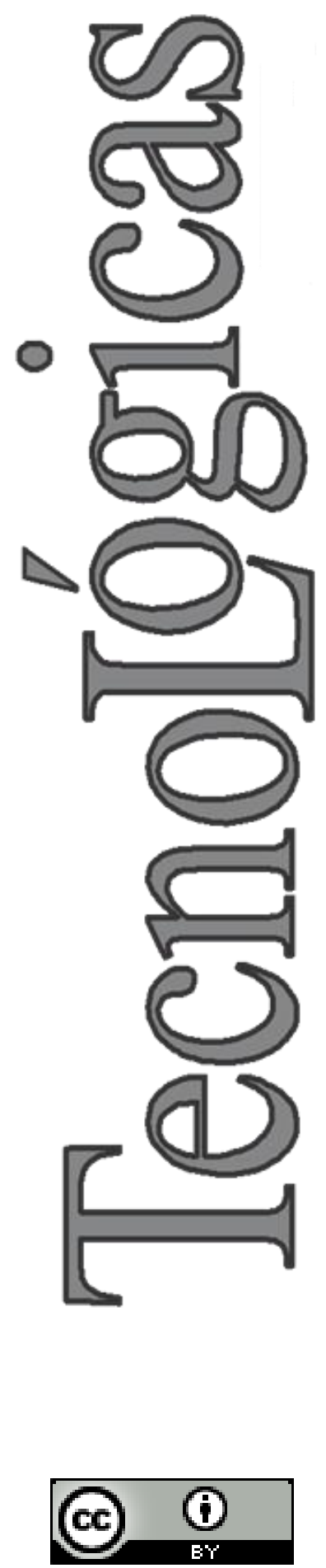

\section{Preparación y caracterización de carbón activado a partir de torta de higuerilla}

\section{Preparation and characterization of activated carbon from castor de-oiled cake}

\author{
Viviana M. Ospina-Guarín ${ }^{1}$, \\ Robison Buitrago-Sierra², Diana P. López-López ${ }^{3}$
}

Recibido: 10 de noviembre de 2013, Aceptado: 20 de diciembre de 2013

Como citar / How to cite

V. M. Ospina-Guarín, R. Buitrago-Sierra y D. P. López-López, "Preparación y caracterización de carbón activado a partir de torta de higuerilla”, Tecno Lógicas, vol. 17, no. 32, pp. 75-84, 2014.

1 Estudiante de Química, Facultad de Ciencias Exactas y Naturales, Química de Recursos Energéticos y Medio Ambiente - QUIREMA, Universidad de Antioquia, Medellín-Colombia, vimaosgu@gmail.com

2 Ph.D. Ciencia de Materiales, Facultad de Ingeniería, Grupo de Investigación en Materiales Avanzados y Energía - MATyER, Instituto Tecnológico Metropolitano, Medellín-Colombia, robinsonbuitrago@itm.edu.co

3 Ph.D. Química, Facultad de Ciencias Exactas y Naturales, Química de Recursos Energéticos y Medio Ambiente - QUIREMA, Universidad de Antioquia, Medellín-Colombia, dplope@gmail.com 


\section{Resumen}

Los residuos de biomasa han sido usados para la producción de carbones activados, en este proceso, el método para obtenerlos y la materia prima determinan propiedades como la porosidad y área superficial del carbón. Tras la extracción del aceite de higuerilla se genera un subproducto sólido (torta) de difícil salida, que fue usado en la producción de carbón activado, en busca de la valorización de este residuo sólido. Para tal fin se emplearon dos métodos tradicionales. En primer lugar, activación física usando como agentes activantes vapor de agua, $\mathrm{CO}_{2}$ y una mezcla de estos gases, adicionalmente se realizó activación química usando $\mathrm{K}_{2} \mathrm{CO}_{3}$ como agente activante. Algunos de los carbones obtenidos fueron caracterizados usando isotermas de adsorción/desorción de $\mathrm{N}_{2}$ donde se lograron áreas superficiales BET entre 255,98 $\left(\mathrm{m}^{2} / \mathrm{g}\right)$ y 1218,43 $\left(\mathrm{m}^{2} / \mathrm{g}\right)$, del análisis por SEM y EDS se encontró que los materiales obtenidos por ambos tipos de activación son principalmente amorfos y que las características morfológicas de los carbones obtenidos mediante activación física son muy diferentes de los obtenidos por activación química. Finalmente, a través de impregnación con sales de $\mathrm{Ni}$ y Mo se evidenció que por características como la alta dispersión obtenida, estos materiales carbonosos presentan un potencial para ser usados como soporte catalítico.

\section{Palabras clave}

Biomasa, carbón activado, materiales porosos, activación física, activación química.

\section{Abstract}

Biomass residues have been used to produce activated carbons. On this process, the activation method and the raw composition determine the properties as porosity and surface area of the charcoal. After the extraction of castor oil, there is a solid byproduct (cake) of low added value, which was used in the production of activated carbon to add value to this waste. For this purpose two traditional methods were used, first, physical activation using as activating agents steam, $\mathrm{CO}_{2}$ and mixture of both, and additionally chemical activation using $\mathrm{K}_{2} \mathrm{CO}_{3}$ as the activating agent. Some activated carbons were characterized using $\mathrm{N}_{2}$ adsorption isotherms, BET surface areas varied between 255.98 $\left(\mathrm{m}^{2} / \mathrm{g}\right)$ and $1218.43\left(\mathrm{~m}^{2} / \mathrm{g}\right)$. By SEM and EDS analysis was possible to observe that materials obtained by the two types of activation are principally amorphous and morphological characteristics of the carbon obtained by physical activation are very different from those obtained by chemical activation. Finally, through impregnation of inorganic phases of $\mathrm{Ni}$ and Mo was revealed that the high dispersion characteristics, these carbonaceous materials will have potential to be used as catalyst support.

\section{Keywords}

Biomass, activated carbon, porous materials, physical activation, chemical activation. 


\section{INTRODUCCIÓN}

Los procesos termoquímicos de conversión como la pirólisis, se han usado sobre la biomasa para recuperación energética [1], [2] y producción de algunos materiales como carbón activado [3], [4] a pesar de que este puede producirse a partir de carbón mineral o coque de petróleo. Se tiene especial interés sobre los residuos de biomasa [5], puesto que son materia prima de bajo costo, además su tratamiento es amigable con el medio ambiente.

Para la producción de carbón activado a partir de biomasa, se distinguen comúnmente dos métodos de activación: activación física y activación química [6], el primero de estos envuelve dos pasos, la pirólisis y la posterior reacción del pirolizado con una atmósfera de gases oxidantes como vapor de agua, $\mathrm{CO}_{2}$ o mezcla de estos [5] a una temperatura entre $800^{\circ} \mathrm{C}$ y $1000^{\circ} \mathrm{C}$ para extraer átomos de carbono de la estructura (1) (2) y favorecer la formación de poros en el material carbonoso [6].

$\mathrm{C}+\mathrm{CO}_{2} \rightarrow 2 \mathrm{CO}$

$\mathrm{C}+\mathrm{H}_{2} \mathrm{O} \rightarrow \mathrm{H}_{2}+\mathrm{COn}$

La activación química, solo involucra un paso, ya que se usan sustancias químicas como $\mathrm{ZnCl}_{2}, \mathrm{H}_{3} \mathrm{PO}_{4}, \mathrm{KOH}$ o $\mathrm{K}_{2} \mathrm{CO}_{3}$ que modifican el proceso de carbonización. Para este, la biomasa se impregna con dichas sustancias y posteriormente es carbonizada a temperaturas entre $400^{\circ} \mathrm{C}$ y $900^{\circ} \mathrm{C}$. Los métodos químicos generan un mayor grado de porosidad en comparación con los métodos físicos [5], al promover reacciones de deshidratación u oxidación en el precursor celulósico [3], [5], y aunque los mecanismos por los cuales se da la formación de poros no son claramente entendidos [7], se ha encontrado por ejemplo, que el $\mathrm{K}_{2} \mathrm{CO}_{3}$ es reducido por el carbón en atmósfera inerte [8] dando paso a la formación de potasio metálico. $\mathrm{El} \mathrm{K}_{2} \mathrm{CO}_{3}$ es de gran interés puesto que no tiene asociados problemas ambientales como el $\mathrm{ZnCl}_{2}$, es de bajo costo y no es corrosivo a diferencia del $\mathrm{KOH}$.

Se han reportado estudios de la producción de carbones activados, usando los métodos mencionados y diferentes fuentes de biomasa como hueso de aceituna [3], cáscara de palma [7], mazorca de maíz [9], residuos de té [10] y muchos más [5], [11]. Por otro lado estos carbones tienen usos diversos, principalmente como adsorbentes [12], pero también pueden usarse como soporte catalítico [13]. Los carbones activados usados en catálisis presentan un volumen especifico de poro entre 0,9 y 1,3 $\mathrm{ml} / \mathrm{g}$ y áreas superficiales entre 800 y 1200 $\mathrm{m}^{2} / \mathrm{g}$ [14]. La gran ventaja del uso de carbones activados como soporte catalítico, además de su bajo costo, es la idea de obtener grandes dispersiones de fases inorgánicas, haciendo más eficiente su uso, al maximizar el área expuesta de las mismas.

Las semillas de higuerilla son una biomasa que puede ser tratada con el fin de producir carbones activados. Tras la extracción del aceite de higuerilla el cual tiene múltiples usos [15], se genera un residuo sólido de difícil salida, debido a la presencia de toxinas como la ricina [16]. En este trabajo se busca dar valor agregado a este residuo sólido, mediante la preparación de carbones activados y la caracterización de algunos de ellos para su potencial uso como soporte catalítico.

\section{METODOLOGÍA}

\subsection{Materiales}

El residuo se obtiene tras la extracción del aceite de higuerilla de unas semillas donadas por Colombiana de Biocombustibles S.A. (variedad BRS-149 Nordestina). Las semillas de nordestina son maceradas y se someten a extracción con soxhlet para la obtención del aceite, de acuerdo al método tradicionalmente usado para este fin [16]. El residuo sólido obtenido es llamado torta de higuerilla. 
Como agente activante, se usó carbonato de potasio anhidro $\left(\mathrm{K}_{2} \mathrm{CO}_{3}\right.$, Merck, pureza $>99 \%$ ). Como precursores de Ni y Mo se usaron nitrato de níquel hexahidratado $\left(\mathrm{Ni}\left(\mathrm{NO}_{3}\right) \cdot 6 \mathrm{H}_{2} \mathrm{O}\right.$, Panreac. Pureza 98\%) y heptamolibdato de amonio tetrahidratado $\left(\left(\mathrm{NH}_{4}\right)_{6} \mathrm{Mo}_{7} \mathrm{O}_{24} \cdot 4 \mathrm{H}_{2} \mathrm{O}\right.$, Panreac. Pureza $99 \%)$.

\subsection{Preparación de los carbones activados por activación física}

Los carbones activados fueron obtenidos mediante pirólisis de la torta de higuerilla y posterior activación del pirolizado. En un horno horizontal, dentro de una cápsula de acero, fue colocada la biomasa que fue pirolizada en atmósfera de nitrógeno a $800^{\circ} \mathrm{C}$ por 1 hora. Luego las muestras fueron enfriadas a temperatura ambiente y fueron tamizadas para homogenizar su tamaño entre $75 \mu \mathrm{m}$ y $180 \mu \mathrm{m}$. Posteriormente para la activación, usando el mismo horno, las muestras fueron llevadas hasta la temperatura de activación en atmósfera de nitrógeno, gas que fue cambiado por el agente activante (nitrógeno saturado con vapor de agua a $80^{\circ} \mathrm{C}, \mathrm{CO}_{2} \mathrm{o}$ $\mathrm{CO}_{2}$ saturado con vapor de agua a $80^{\circ} \mathrm{C}$ ) evaluando el efecto de diferentes temperaturas y tiempos de activación [5], [17]. Finalmente las muestras fueron enfriadas en atmósfera de nitrógeno.

\subsection{Preparación de los carbones activados por activación química}

Para la activación química, la biomasa fue molida y tamizada con malla 30 (0.6 $\mathrm{mm}$ ), luego fue impregnada con una solución de carbonato de potasio teniendo en cuenta una relación de peso $\mathrm{K}_{2} \mathrm{CO}_{3}$ : biomasa, de 1:1 y $2: 1$ por un periodo de tiempo de 24 horas a temperatura ambiente, posteriormente la muestra fue secada en estufa a $110^{\circ} \mathrm{C}$. Finalmente, la muestra fue carbonizada a $800^{\circ} \mathrm{C}$ por 1 hora en atmósfera de $\mathrm{N}_{2}$, después de enfriarse fue lavada con agua destilada hasta $\mathrm{pH}$ neutro [8]-[10].

\subsection{Impregnación húmeda}

Mediante impregnación húmeda, utilizando un rotavapor por $14 \mathrm{~h}$ se dispersaron sobre el carbón activado fases de $\mathrm{Ni}$ y Mo, utilizando como sales precursoras $\mathrm{Ni}\left(\mathrm{NO}_{3}\right) \cdot 6 \mathrm{H}_{2} \mathrm{O}$ y $\left(\mathrm{NH}_{4}\right)_{6} \mathrm{Mo}_{7} \mathrm{O}_{24} \cdot 4 \mathrm{H}_{2} \mathrm{O}$, en las cantidades apropiadas para obtener un $10 \%$ en peso de Mo y $2 \%$ de Ni en la superficie del soporte. Después de la impregnación el exceso de volumen fue eliminado por evaporación en vacío durante $3 \mathrm{~h}$, posteriormente las muestras fueron llevadas a la estufa $\left(90^{\circ} \mathrm{C}\right)$ por $12 \mathrm{~h}$. Finalmente, para descomponer los precursores las muestras fueron calcinadas a $500^{\circ} \mathrm{C}$ por $1 \mathrm{~h}$ en atmósfera de $\mathrm{N}_{2}$ [18],[19].

\subsection{Caracterización}

Para la caracterización de la biomasa se realizó análisis próximo usando un TGA Q500 marca TA Instruments. El análisis termogravimétrico de la torta de higuerilla fue realizado a una velocidad de calentamiento de $40^{\circ} \mathrm{C} /$ minuto en atmósfera de nitrógeno. Finalmente a $800^{\circ} \mathrm{C}$ el nitrógeno fue sustituido por aire, y la combustión del carbono fijo da paso a la formación de cenizas.

El análisis elemental (CHNSO) fue efectuado en un equipo marca LECO, modelo Truspec, la composición inorgánica de la biomasa se determinó con un espectrómetro de fluorescencia de rayos $\mathrm{X}$ marca THERMO modelo OPTIM'X. El análisis de área superficial fue realizado en un analizador de área superficial y porosidad ASAP 2020 marca Micrometrics. La morfología de las muestras fue evaluada en alto vacío y con las señales de electrones secundarios y retrodispersados, usando un Microscopio Electrónico de Barrido marca JEOL JSM6490 LV. 


\section{RESULTADOS Y DISCUSIÓN}

\subsection{Caracterización de la torta de higuerilla}

El análisis termogravimétrico de la torta de higuerilla se muestra en la Fig. 1. Este presenta varias etapas de pérdida de peso; la primera está asociada a la humedad $\left(90^{\circ} \mathrm{C}\right)$, alrededor de $200^{\circ} \mathrm{C}$ inicia la descomposición del material que da paso a la formación de $\mathrm{CO}, \mathrm{CO}_{2}$ y una gran variedad de compuestos volátiles, como alcoholes, ácidos, fenoles, entre otros, que se forman tras el rompimiento de los enlaces químicos de la celulosa, hemicelulosa, lignina y ácidos grasos presentes en la biomasa [20]. Alrededor de $600^{\circ} \mathrm{C}$ la mayor parte del material se ha descompuesto dejando como residuo el carbono fijo, que corresponde a la organización del material carbonoso no volátil de la biomasa, la presencia de heteroátomos como oxígeno, nitrógeno y azufre, hace que este pueda presentar una gran variedad de grupos funcionales.

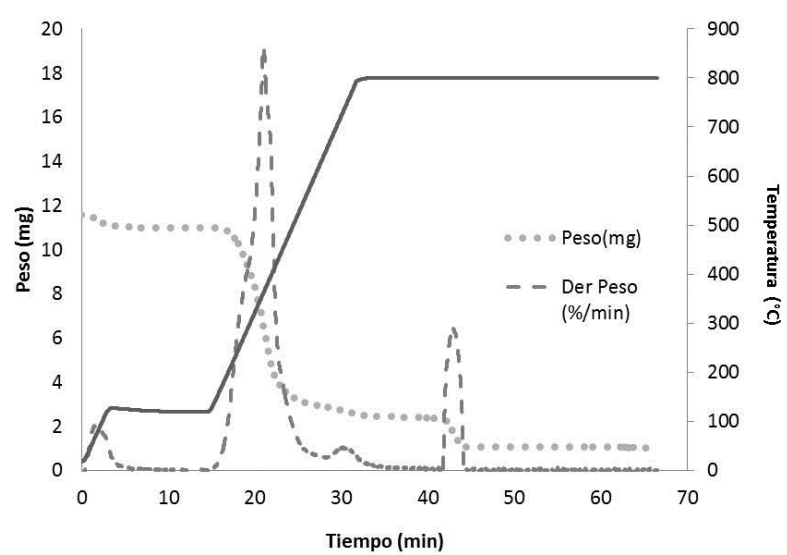

Fig. 1. Análisis termogravimétrico de la torta de higuerilla. Fuente: Autores

Los resultados correspondientes al análisis próximo y análisis elemental hechos en base húmeda, de la muestra de la torta de higuerilla, se presentan en la Tabla 1, en esta se puede ver el alto contenido de oxígeno de la muestra, característico de una biomasa ya que esta tiene un alto contenido de polisacáridos como la celulosa y hemicelulosa [21]; adicionalmente un alto contenido de nitrógeno por la presencia de algunas proteínas, principalmente de ricina [16]. El análisis próximo muestra características típicas de la biomasa, un alto contenido de material volátil, compuesto principalmente por hidrocarburos livianos y alquitranes [22].

Tabla 1. Propiedades de la torta de higuerilla. Fuente: Autores

\begin{tabular}{lccc}
\hline $\begin{array}{l}\text { Análisis } \\
\text { elemental }\end{array}$ & $\% \mathrm{p} / \mathrm{p}$ & Análisis próximo & $\% \mathrm{p} / \mathrm{p}$ \\
\hline $\mathrm{C}$ & 52,36 & Humedad & 5,07 \\
$\mathrm{~N}$ & 4,75 & Material volátil & 74,45 \\
$\mathrm{O}$ & 34,36 & Carbono fijo & 11,23 \\
$\mathrm{H}$ & 7,19 & Cenizas & 8,97 \\
$\mathrm{~S}$ & 0,09 & & \\
\hline
\end{tabular}

El contenido de cenizas, que puede variar dependiendo del tipo de biomasa y de las condiciones del suelo donde se cultive, es parte integral de las plantas y consiste en un amplio rango de material mineral como sales de calcio, potasio, silicio y magnesio, que hacen parte de la estructura de la biomasa [22]. Mediante un análisis de fluorescencia de rayos $\mathrm{X}$, se determinó la composición inorgánica del material a la biomasa fresca, estos datos se muestran en la Tabla 2. En la producción de carbón activado como soporte catalítico es de gran importancia determinar el contenido materia mineral del mismo, ya que la presencia de metales como hierro, níquel y molibdeno podría tener cierta actividad catalítica intrínseca para algunas reacciones [23], entre ellas las reacciones de Hidrodesoxigenación (HDO) en las cuales se pretenden usar estos materiales en trabajos posteriores, pero como se ve de la Tabla 2, la biomasa tratada en este trabajo, presenta un bajo contenido de los mismos.

\subsection{Optimización del método para la obten- ción del carbón activado}

Las características del carbonizado obtenido después de la pirólisis se presentan en la Tabla 3 , en comparación con la bio- 
masa, el carbonizado presenta un lógico incremento en el contenido de carbono, y una consecuente disminución del oxígeno y el hidrógeno, fruto del tratamiento térmico.

Tabla 2: Resultados del análisis de fluorescencia de rayos X de la torta de higuerilla. Fuente: Autores

\begin{tabular}{cccccccc}
\hline Óxido & $\mathrm{P}_{2} \mathrm{O}_{5}$ & $\mathrm{CaO}$ & $\mathrm{K}_{2} \mathrm{O}$ & $\mathrm{MgO}$ & $\mathrm{SO}_{3}$ & $\mathrm{Fe}_{2} \mathrm{O}_{3}$ & $\mathrm{Na}_{2} \mathrm{O}$ \\
$\%$ p/p & 6,210 & 5,780 & 2,260 & 1,720 & 0,954 & 0,171 & 0,140 \\
\hline Óxido & $\mathrm{Al}_{2} \mathrm{O}_{3}$ & $\mathrm{ZnO}$ & $\mathrm{MnO}$ & $\mathrm{SiO}_{2}$ & $\mathrm{CuO}$ & $\mathrm{Cr}_{2} \mathrm{O}_{3}$ & $\mathrm{NiO}$ \\
$\%$ p/p & 0,106 & 0,048 & 0,034 & 0,033 & 0,012 & 0,007 & 0,004 \\
\hline Óxido & $\mathrm{ZrO}_{2}$ & $\mathrm{BaO}$ & $\mathrm{SnO}$ & $\mathrm{TiO}_{2}$ & $\mathrm{MoO}_{3}$ & $\mathrm{LOI}$ & \\
$\%$ p/p & 0,003 & 0,002 & 0,002 & 0,002 & 0,002 & 85,504 & \\
\hline
\end{tabular}

Tabla 3. Análisis elemental del carbonizado. Fuente: Autores

\begin{tabular}{lc}
\hline Análisis Elemental & $\% \mathrm{p} / \mathrm{p}$ \\
\hline $\mathrm{C}$ & 65,81 \\
$\mathrm{~N}$ & 3,85 \\
$\mathrm{O}$ & 10,02 \\
$\mathrm{H}$ & 1,12 \\
$\mathrm{~S}$ & 0,26 \\
\hline
\end{tabular}

\subsection{Activación física}

El objetivo de activar el material carbonoso obtenido en la pirólisis es: modificar la porosidad del carbón, incrementar el volumen de poros y su área superficial [6]; por ello después de macerar y tamizar para lograr un tamaño de partícula homogéneo entre 80 y $150 \mu \mathrm{m}$, se procedió a la activación del carbonizado de higuerilla. La activación que se evaluó en primer lugar fue con vapor de agua a diferentes condiciones: $800^{\circ} \mathrm{C}$ por 1 hora (CA81) y por 2 horas (CA82); a $900^{\circ} \mathrm{C}$ por 1 y 2 horas (CA91, CA92). Los datos obtenidos se presentan en la Tabla 4, para ambas temperaturas se observa que un mayor tiempo de reacción aumenta los porcentajes de pérdida y favorece el aumento del área superficial de las muestras, el hecho de aumentar la temperatura de reacción, también incrementó el porcentaje de pérdida y las áreas superficiales.

Debido a que se busca un carbón activado que pueda ser utilizado como soporte catalítico, respecto a las áreas superficia- les, bajo las condiciones más drásticas $\left(900^{\circ} \mathrm{C} / 2 \mathrm{~h}\right)$ se logró un área de $462 \mathrm{~m}^{2} / \mathrm{g}$ que es apenas la mitad del área que propone la literatura para ser usado con este fin [14]. Por esto, se decidió realizar una activación usando un agente activante más agresivo como el $\mathrm{CO}_{2}$ [6], los datos de conversión y área se muestran en la Tabla 4, de igual manera que con vapor de agua, se hace la activación a $800^{\circ} \mathrm{C}$ por 1 hora (CAC81) y a $900^{\circ} \mathrm{C}$ por 2 horas (CA92). Cuando se retiró la muestra tratada a $900^{\circ} \mathrm{C}$ por dos horas, se observó que el carbón tenía pequeñas partículas blancas asociadas con cenizas, lo que no sucedió con la muestra a $800^{\circ} \mathrm{C}$, esto es debido a que la activación bajo esas condiciones es muy drástica y gasifica en gran medida el material, hecho que se corrobora al determinar que el porcentaje de cenizas en la muestra es del $46,46 \%$, por tanto estas condiciones no son las más apropiadas para producir un carbón activado que pueda ser utilizado como soporte catalítico.

\begin{tabular}{lcccc}
\multicolumn{4}{c}{$\begin{array}{c}\text { Tabla 4. Resultados de porcentaje de pérdida, área superfi- } \\
\text { cial y porcentaje de cenizas para las muestras activadas } \\
\text { físicamente. Fuente: Autores }\end{array}$} \\
$\begin{array}{l}\text { Tipo de } \\
\text { activación }\end{array}$ & Condiciones & $\begin{array}{c}\text { Porcentaje } \\
\text { pérdida }\end{array}$ & $\begin{array}{c}\text { Área } \\
\text { superficial } \\
\left(\mathrm{m}^{2} / \mathrm{g}\right)\end{array}$ & $\begin{array}{c}\text { Porcentaje } \\
\text { cenizas }\end{array}$ \\
\hline & CA81 & 16,63 & 255,98 & n.d \\
Activación & CA82 & 25,62 & 341,94 & n.d \\
con $\mathrm{H}_{2} \mathrm{O}$ & CA91 & 23,56 & 336,51 & n.d \\
& CA92 & 45,11 & 462,44 & 23 \\
\hline $\begin{array}{l}\text { Activación } \\
\text { con } \mathrm{CO}_{2}\end{array}$ & CAC81 & 19,9 & 258,81 & n.d \\
\hline & CAC92 & 71,16 & 879,02 & 46,46 \\
\hline $\begin{array}{l}\text { Activación } \\
\text { con }\end{array}$ & CAAC81 & 28,4 & 406,82 & n.d \\
$\mathrm{H}_{2} \mathrm{O} / \mathrm{CO}_{2}$ & CAAC83 & 56,62 & 565,74 & 22,73 \\
& CAAC852 & 73,05 & 655,01 & 52,47 \\
\hline
\end{tabular}

n.d: no determinado

Otro procedimiento que ha sido propuesto para activar carbón, es el uso de una mezcla de $\mathrm{CO}_{2} / \mathrm{H}_{2} \mathrm{O}_{\text {(vapor) }}$ [5], estas condiciones a más baja temperatura pueden ser apropiadas para aumentar el área superficial del carbón, por esto se procedió a 
realizar una activación a $800^{\circ} \mathrm{C}$ por 1,2 y 3 horas (CAAC81, CAAC82, CAAC83) y a $850^{\circ} \mathrm{C}$ por 2 horas (CAAC852). Con la activación hecha a $850^{\circ} \mathrm{C}$ nuevamente se obtuvo un carbón con alto contenido de cenizas (ver Tabla 4). De lo anterior se encuentra que la muestra tratada a $800^{\circ} \mathrm{C}$ por 2 horas con la mezcla de $\mathrm{CO}_{2}$ y $\mathrm{H}_{2} \mathrm{O}$, presenta las mejores características en cuanto a contenido en cenizas y área superficial. Todos los resultados de la activación física se muestran en la Tabla 4.

Aunque de los experimentos realizados con la mezcla de $\mathrm{CO}_{2} / \mathrm{H}_{2} \mathrm{O}$ se obtuvieron carbones con una mayor área superficial, esta sigue siendo insuficiente con respecto a lo reportado [14], por lo que se realizaron experimentos de activación química usando carbonato de potasio como agente activante [8].

\subsection{Activación química}

Se prepararon carbones activados mediante un método químico, usando como agente activante $\mathrm{K}_{2} \mathrm{CO}_{3}$; se realizaron dos ensayos en los que: CAK181 corresponde al carbón obtenido tras la impregnación con una relación en peso de 1:1 $\left(\mathrm{K}_{2} \mathrm{CO}_{3} /\right.$ biomasa) y CAK281 corresponde al carbón obtenido con una de impregnación en relación de $2: 1$.

Los resultados de área superficial obtenidos se muestran en la Tabla 5, las áreas superficiales obtenidas son mucho mayores respecto a las encontradas para la activación física; valores de área superficial que colocan el material dentro de un rango que posibilita su uso en aplicaciones como soporte catalítico [14], se observa que además el contenido de cenizas en el carbón CAK181 es menor, lo que era de esperarse al tener una mayor concentración de agente activante el CAK281. Tras obtener un área superficial mayor para la muestra CAK181 y un contenido de cenizas menor, se elige esta muestra para el análisis de morfología de la superficie.
Tabla 5. Resultados para las muestras activadas químicamente. Fuente: Autores

\begin{tabular}{lcc}
\hline Condiciones & Área superficial $\left(\mathrm{m}^{2} / \mathrm{g}\right)$ & \% Cenizas \\
\hline CAK181 & 1218,43 & 7,55 \\
CAK281 & 1197,96 & 23,63 \\
\hline
\end{tabular}

\subsection{Morfología de la superficie}

La morfología de los carbones que mostraron mejores características de área superficial, se estudió mediante microscopía electrónica de barrido. Se muestran las micrografías de los carbones activados Fig. 2 obtenidos mediante activación física $\mathrm{y}$ mediante activación química respectivamente que presentaron mejores áreas superficiales, contrastado con el porcentaje de cenizas (CAAC82 y CAK181). Como se puede ver de las micrografías, el carbón activado obtenido con activación física CAAC82 (Fig. 2a y 2b), es un material muy homogéneo, de partículas con caras planas, en el que la porosidad no es muy perceptible. Por otro lado el material obtenido por la activación química (Fig. 2c y 2d), presenta una estructura heterogénea, haciéndose evidente la porosidad del mismo por la irregularidad de sus partículas, se puede ver claramente una estructura más porosa, lo que está de acuerdo con los estudios de área superficial realizados para el material.
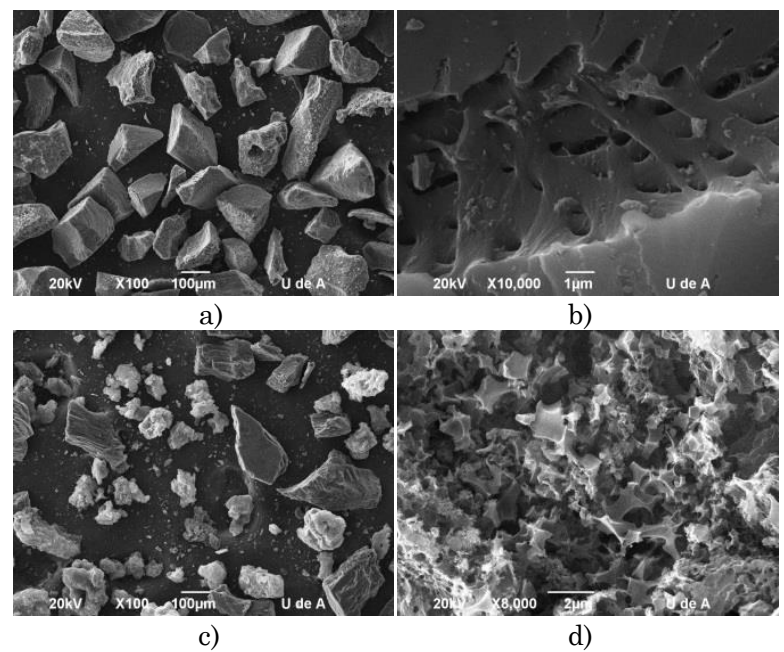

Fig. 2. Micrografías SEM de los carbones activados CAAC82 (a y b); CAK181 (c y d). Fuente: Autores 
Como se ha mencionado anteriormente, una de las posibles aplicaciones para estos materiales, es su uso como soportes catalíticos [6], [13], [24]; teniendo en cuenta que en el diseño de catalizadores la buena dispersión de las fases inorgánicas sobre los soportes es un requerimiento, es fundamental evaluar la dispersión de algunas sales sobre los carbones activados. Para este fin, mediante impregnación húmeda se depositaron sales de Ni y Mo [25], [26] sobre los carbones CAAC82 y CAK181. En la Fig. 3, se muestran las micrografías de estos materiales, una vez realizada la descomposición de los precursores. En estas imágenes, se observa que las fases de $\mathrm{Ni} \mathrm{y}$ Mo están apropiadamente dispersas sobre el material carbonoso, no se aprecian aglomerados o zonas de bajo contenido mineral. De las ampliaciones se aprecia cómo debido a la morfología del material, los metales se dispersan de mejor manera sobre el carbón preparado por activación química.
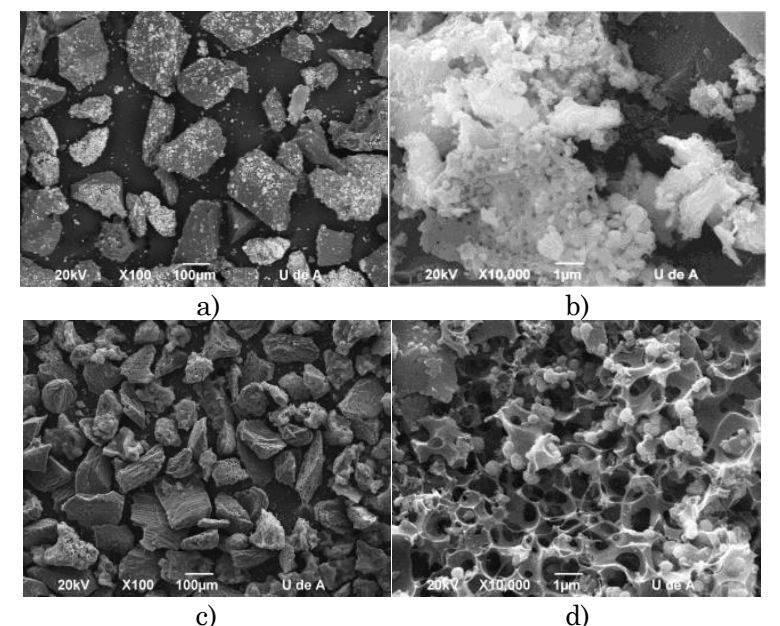

Fig. 3. Micrografía SEM del carbón activado CAAC82 (a y b) y CAK181 (c y d) impregnados con una fase inorgánica compuesta por Ni y Mo. Fuente: Autores

Adicionalmente, a ambas muestras se les realizó un estudio de SEM-EDS, con el fin de estudiar la composición química en la superficie de los carbones (Fig. 4). En estos se puede ver principalmente que se caracteriza por la presencia de $\mathrm{Ca}, \mathrm{Mg}$ y $\mathrm{O}$, como es característico de la biomasa; ade- más, los metales que se soportaron ( $\mathrm{Ni} \mathrm{y}$ Mo) se encontraron de manera semicuantitativa en una proporción de $2,74 \%$ en $\mathrm{Ni}$ y de $8,77 \%$ en Mo para CAAC 82 ; y $1,75 \%$ y $8,24 \%$ respectivamente para CAK181, valores que están de acuerdo con lo que teóricamente se proponía soportar en el material carbonoso.
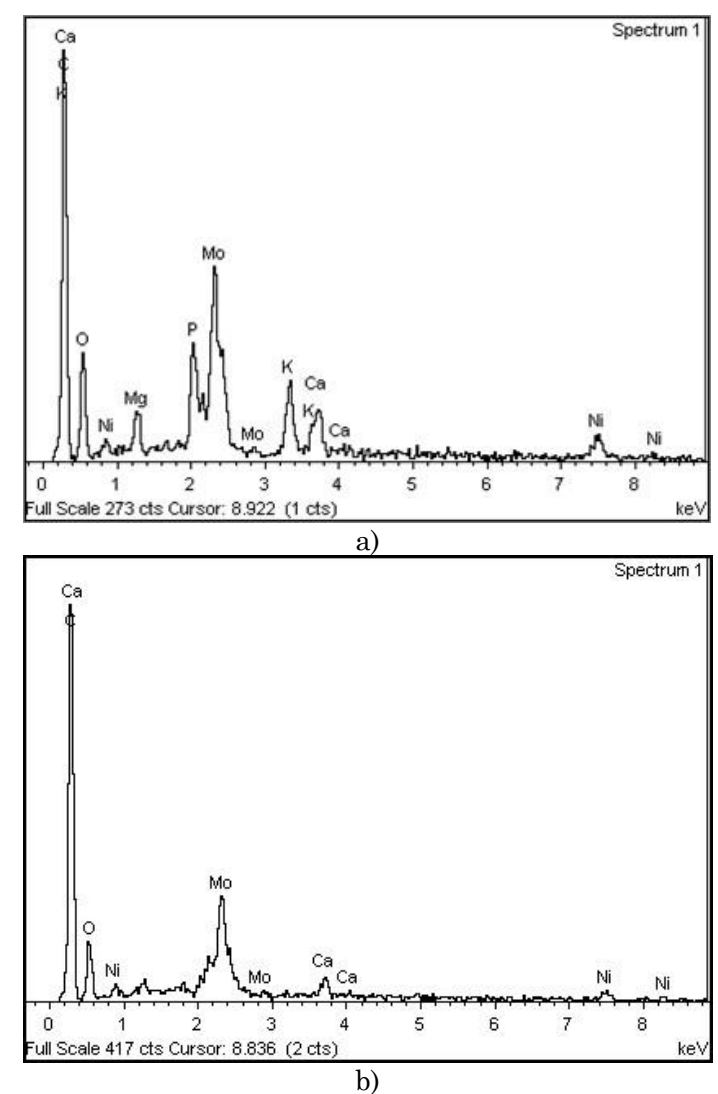

Fig. 4. Análisis elemental en la superficie (EDS) de los carbones activados CAAC82 (a) y CAK181 (b) impregnados con sales de Mo y Ni. Fuente: Autores

La dispersión de las fases inorgánicas se observó además por mapeo de composición química en el SEM, en ésta es posible determinar la dispersión de cada metal. En la Fig. 5 se muestran las imágenes de mapeo para las muestras de CAAC82 y CAK181, de los metales más abundantes. Se puede observar que aunque ambos metales (Ni y Mo), muestran una excelente dispersión sobre toda la superficie del material carbonoso, sin formación de aglomerados o zonas con escazes de alguno de los metales, en el 
carbón CAK181, se aprecia una mayor dispersión de ambos metales, evidenciada en la falta de zonas con una mayor densidad de los puntos referentes a la presencia de cada metal, generando mapas de dispersión mucho mas homogéneos para ambos metales.

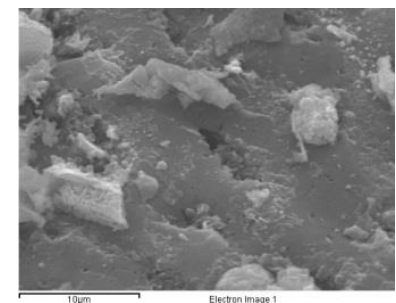

a)

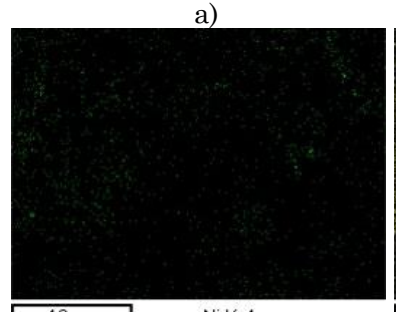

c)

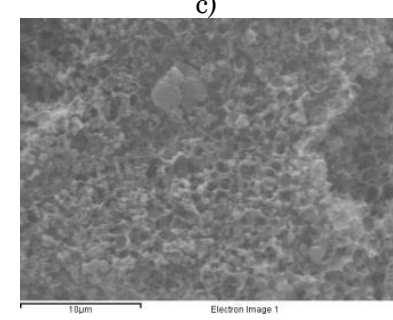

e)

g)

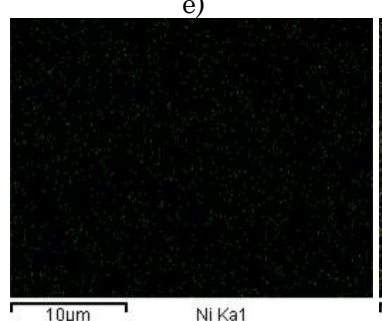

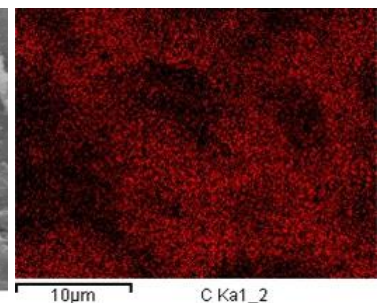

b)

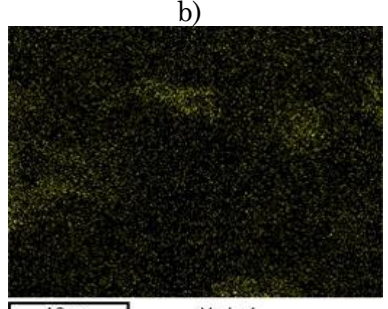

d)

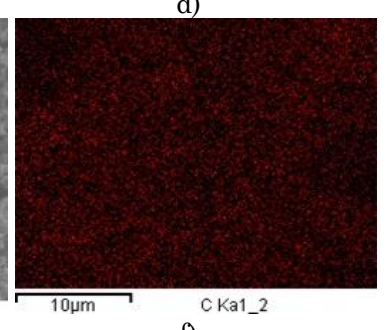

f)

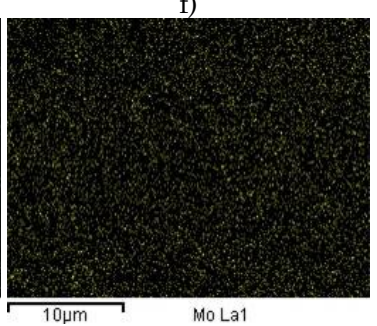

h)
Fig. 5. Mapas de composición elemental SEM-EDS de los carbones activados CAAC82 (a, b, c y d) y CAK181 (e, f, g y h) impregnados con un fase metálica de Mo y Ni. Fuente: Autores

\section{CONCLUSIONES}

Se prepararon carbones activados del pirolizado de torta de higuerilla mediante activación física con vapor de agua, $\mathrm{CO}_{2} \mathrm{y}$ mezcla de estos. De las condiciones utilizadas los mejores resultados con un área superficial de $565,74 \mathrm{~m}^{2} / \mathrm{g}$ y bajo contenido de cenizas, se obtuvieron para el carbón activado con la mezcla de $\mathrm{CO}_{2} / \mathrm{H}_{2} \mathrm{O}$ por 2 horas. Adicionalmente se prepararon carbones activados por métodos químicos usando como agente activante $\mathrm{K}_{2} \mathrm{CO}_{3}$, se encontró que esta última favorece la formación de mayores áreas superficiales en el material, lográndose un valor máximo de $1218,43 \mathrm{~m}^{2} / \mathrm{g}$ para una relación en masa de 1:1 (biomasa/ $\mathrm{K}_{2} \mathrm{CO}_{3}$ ), se encontró además que el contenido de cenizas es menor en los carbones activados químicamente. Finalmente, se corroboró el potencial uso de estos materiales como soporte catalítico, al observar la alta dispersión de las fases inorgánicas depositadas sobre el carbón activado, sin formación de aglomerados o sinterizados.

\section{AGRADECIMIENTOS}

Los autores agradecen al programa sostenibilidad 2013-2014 de la Universidad de Antioquia.

\section{REFERENCIAS}

[1] N. Abdullah, F. Sulaiman, and H. Gerhauser, "Characterisation of Oil Palm Empty Fruit Bunches for Fuel Application,” J. Phys. Sci., vol. 22, no. 1, pp. 1$24,2011$.

[2] A. V. Bridgwater, "Review of fast pyrolysis of biomass and product upgrading," Biomass and Bioenergy, vol. 38, pp. 68-94, Mar. 2012.

[3] J. F. González, S. Román, J. M. Encinar, and G. Martínez, "Pyrolysis of various biomass residues and char utilization for the production of activated carbons," J. Anal. Appl. Pyrolysis, vol. 85, no. 1-2, pp. 134-141, May 2009.

[4] J. Hayashi, A. Kazehaya, K. Muroyama, and A. P. Watkinson, "Preparation of activated carbon from lignin by chemical activation," Carbon N. Y., vol. 38, no. 13, pp. 1873-1878, Jan. 2000.

[5] O. Ioannidou and A. Zabaniotou, "Agricultural residues as precursors for activated carbon production - A review," Renew. Sustain. Energy Rev., vol. 11, no. 9, pp. 1966-2005, Dec. 2007.

[6] H. Marsh and F. Rodriguez-Reinoso, Activated Carbon. Elsevier Science \& Technology Books, 2006, p. 554.

[7] D. Adinata, W. M. A. Wan Daud, and M. K. Aroua, "Preparation and characterization of activated carbon from palm shell by chemical activation with K2CO3.," Bioresour. Technol., vol. 98, no. 1, pp. 145-9, Jan. 2007. 

torta de higuerilla

[8] J. Hayashi, T. Horikawa, I. Takeda, K. Muroyama, and F. Nasir Ani, "Preparing activated carbon from various nutshells by chemical activation with $\mathrm{K} 2 \mathrm{CO} 3$," Carbon N. Y., vol. 40, no. 13, pp. 2381-2386, Jan. 2002.

[9] M. Song, B. Jin, R. Xiao, L. Yang, Y. Wu, Z. Zhong, and Y. Huang, "The comparison of two activation techniques to prepare activated carbon from corn cob," Biomass and Bioenergy, vol. 48, pp. 250-256, Jan. 2013.

[10] I. I. Gurten, M. Ozmak, E. Yagmur, and Z. Aktas, "Preparation and characterisation of activated carbon from waste tea using K2CO3," Biomass and Bioenergy, vol. 37, pp. 73-81, Feb. 2012.

[11] E. Schröder, K. Thomauske, C. Weber, A. Hornung, and V. Tumiatti, "Experiments on the generation of activated carbon from biomass," J. Anal. Appl. Pyrolysis, vol. 79, no. 1-2, pp. 106-111, May 2007.

[12] V. Hernández and A. Bonilla, Lignocellulosic Precursors Used in the Synthesis of Activated Carbon Characterization Techniques and Applications in the Wastewater Treatment. Croatia: InTech, 2012.

[13] P. Serp and J. L. Figueredo, Carbon Materials for catalysis. John Wiley \& Sons, Inc., 2009.

[14] E. Auer, A. Freund, J. Pietsch, and T. Tacke, "Carbons as supports for industrial precious metal catalysts," Appl. Catal. A Gen., vol. 173, no. 2, pp. 259-271, Oct. 1998.

[15] D. S. Ogunniyi, "Castor oil: a vital industrial raw material," Bioresour. Technol., vol. 97, no. 9, pp. 108691, Jun. 2006.

[16] D. J. Barnes, B. S. Baldwin, and D. a. Braasch, "Ricin accumulation and degradation during castor seed development and late germination," Ind. Crops Prod., vol. 30, no. 2, pp. 254-258, Sep. 2009.

[17] E. Apaydın-Varol and A. E. Pütün, "Preparation and characterization of pyrolytic chars from different biomass samples," J. Anal. Appl. Pyrolysis, vol. 98, pp. 29-36, Nov. 2012.
[18] C. Perego and P. Villa, "Catalyst preparation methods," Catal. Today, vol. 34, no. 3-4, pp. 281-305, Feb. 1997.

[19] M. Campanati, G. Fornasari, and A. Vaccari, "Fundamentals in the preparation of heterogeneous catalysts," Catal. Today, vol. 77, no. 4, pp. 299-314, Jan. 2003.

[20] G. W. Huber, S. Iborra, and A. Corma, "Synthesis of transportation fuels from biomass: chemistry, catalysts, and engineering.," Chem. Rev., vol. 106, no. 9, pp. 4044-98, Sep. 2006.

[21] M. Balat, M. Balat, E. Kırtay, and H. Balat, "Main routes for the thermo-conversion of biomass into fuels and chemicals. Part 1: Pyrolysis systems," Energy Convers. Manag., vol. 50, no. 12, pp. 3147-3157, Dec. 2009.

[22] R. K. Singh and K. P. Shadangi, "Liquid fuel from castor seeds by pyrolysis," Fuel, vol. 90, no. 7, pp. 2538-2544, Jul. 2011.

[23] Z. He and X. Wang, "Hydrodeoxygenation of model compounds and catalytic systems for pyrolysis bio-oils upgrading," Catal. Sustain. Energy, vol. 1, pp. 28-52, Jan. 2012.

[24] M. J. Lázaro, M. E. Gálvez, S. Artal, J. M. Palacios, and R. Moliner, "Preparation of steam-activated carbons as catalyst supports," J. Anal. Appl. Pyrolysis, vol. 78, no. 2, pp. 301-315, Mar. 2007.

[25] A. Calafat, "Effect of Surface Oxidation of the Support on the Thiophene Hydrodesulfurization Activity of Mo, $\mathrm{Ni}$, and NiMo Catalysts Supported on Activated Carbon," J. Catal., vol. 162, no. 1, pp. 20-30, Aug. 1996.

[26] P. E. Ruiz, B. G. Frederick, W. J. De Sisto, R. N. Austin, L. R. Radovic, K. Leiva, R. García, N. Escalona, and M. C. Wheeler, "Guaiacol hydrodeoxygenation on MoS2 catalysts: Influence of activated carbon supports," Catal. Commun., vol. 27, pp. 44-48, Oct. 2012. 